\title{
UNA UNIVERSIDAD QUE APRENDE: FORMACIÓN Y EVALUACIÓN DOCENTE EN RED
}

A UNIVERSITY THAT LEARNS:

HIGHER TEACHER EDUCATION AND EVALUATION NETWORK

\section{Volumen 12, Número 1}

Enero-Abril

pp. 1-27

Este número se publicó el 28 de febrero de 2012

\author{
Eleonora Badilla Saxe \\ Silvia Chacón Ramírez \\ Susan Francis Salazar
}

Revista indizada en $\underline{\text { REDALYC }}$

Revista distribuida en las bases de datos:

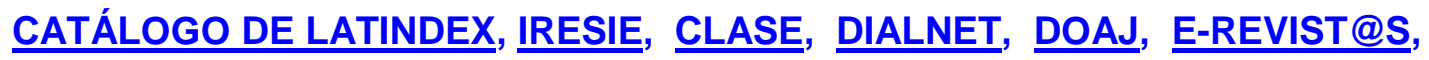

Revista registrada en los directorios:

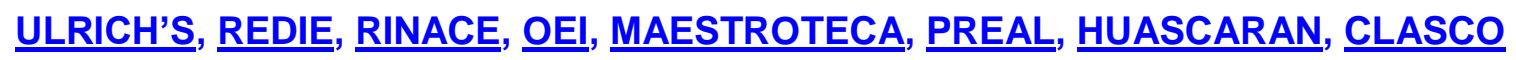




\title{
UNA UNIVERSIDAD QUE APRENDE: FORMACIÓN Y EVALUACIÓN DOCENTE EN RED \\ A UNIVERSITY THAT LEARNS: HIGHER TEACHER EDUCATION AND EVALUATION NETWORK
}

\author{
Eleonora Badilla Saxe ${ }^{1}$ \\ Silvia Chacón Ramírez \\ Susan Francis Salazar ${ }^{3}$
}

\begin{abstract}
Resumen: El presente artículo ofrece, por parte de su Comité Ejecutivo una sistematización sobre la evolución de la Red Institucional de Formación y Evaluación Docente (RIFED). Esta Red es una iniciativa de la Vicerrectoría de Docencia de la Universidad de Costa Rica que ha sido conceptualizada y desarrollada con el fin de apoyar al profesorado universitario para que asuma una nueva mirada a la docencia universitaria en el siglo XXI. Se ofrece un marco contextual y conceptual que intenta responder las interrogantes: ¿por qué una red y no un centro? ¿Para qué evaluar en la UCR? ¿Para qué formar al personal docente la UCR? Entre los logros de la sistematización, se destaca la ruta de aprendizaje de RIFED, una reconceptualización de funciones, así como de los términos formación y evaluación, para finalmente ofrecer una visión prospectiva para RIFED, bajo el título ¿Cómo se mira RIFED en el horizonte?
\end{abstract}

Palabras clave: EDUCACIÓN SUPERIOR, FORMACIÓN DOCENTE UNIVERSITARIA, EVALUACIÓN DOCENTE UNIVERSITARIA, RED, APRENDIZAJE.

\begin{abstract}
This article presents, by its Executive Committee, a systematization of the Institutional Network for Higher Teacher Education and Evaluation RIFED, at the University of Costa Rica UCR, an initiative that is part of the Academic VicePresident's office. RIFED has been conceptualized and implemented with the objective of supporting faculty on assuming a new vision on Higher Education Teaching and Evaluation, relevant to the XXI Century. A conceptual framework aims to answer these questions: Why a network instead of a Center? Why evaluate faculty at UCR? Why educate faculty at UCR? Amongst RIFED's learning path some of its achievements are highlighted: a re-conceptualization of its functions as well as of the terms education and evaluation. Finally it offers a prospective vision of RIFED under the sub-title: How does RIFED appear in the Horizon?
\end{abstract}

Key words: HIGHER EDUCATION; HIGHER EDUCATION TEACHING AND LEARNING, HIGHER EDUCATION FACULTY EVALUATION; HIGHER EDUCATION NETWORKING; LEARNING

\footnotetext{
${ }^{1}$ Doctora en Educación con énfasis en Mediación Pedagógica por la Universidad La Salle. Máster en Educación con énfasis en Tecnologías Digitales por la Universidad de Hartford. Catedrática de la Escuela de Artes Dramáticas de la Universidad de Costa Rica. Directora del Centro de Evaluación Académica. Dirección electrónica: eleonora.badilla@ucr.ac.cr

2 Educadora. Máster en Planificación Curricular de la Universidad de Costa Rica. Directora de la Unidad de Apoyo a la Docencia Mediada con Tecnologías de la Información y Comunicación (METICS) de la Vicerrectoría de Docencia. Profesora de la Escuela de Formación Docente de la Facultad de Educación. Universidad de Costa Rica. Profesora Asociada de la Universidad de Costa Rica. Dirección electrónica: silvia.chacon@ucr.ac.cr

3 Doctora en Educación de la Universidad de Costa Rica, Directora del Departamento de Docencia Universitaria DEDUN; Profesora Asociada de la Universidad de Costa Rica. Dirección electrónica: susan.francis@ucr.ac.cr
}

Artículo recibido: 16 de setiembre, 2011

Aprobado: 16 de febrero, 2012 


\section{Introducción}

A más de tres años de la creación de la Red Institucional de Formación y Evaluación Docente (RIFED) ${ }^{4}$, en la Universidad de Costa Rica (UCR), su comité ejecutor ha considerado la necesidad de sistematizar su desarrollo, por cuanto esta Red representa una de las propuestas más innovadoras por parte de la Vicerrectoría de Docencia de esta Universidad.

Nos referimos a una sistematización dado que se parte de una práctica, la práctica de RIFED, la cual se ordena, evalúa e interpreta, para llegar a una reflexión y producción de conocimiento (Ruiz, 2001).

Jara (2001) se refiere a la sistematización como un proceso histórico y complejo, que ocurre en un momento institucional del cual se es parte. Desde esta perspectiva

Sistematizar experiencias significa entonces entender por qué ese proceso se está desarrollando de esa manera, entender e interpretar lo que está aconteciendo, a partir de un ordenamiento y reconstrucción de lo que ha sucedido en dicho proceso. Por lo tanto, en la sistematización de experiencias, partimos de hacer una reconstrucción de lo sucedido y un ordenamiento de los distintos elementos objetivos y subjetivos que han intervenido en el proceso, para comprenderlo, interpretarlo y así aprender de nuestra propia práctica. El dilema está en no quedarnos sólo en la reconstrucción de lo que sucede sino pasar a realizar una interpretación crítica (Jara, 2001, p.2).

La sistematización sirve entonces para aprender críticamente, con miras a mejorar la propia práctica, compartir con otros experiencias similares, y contribuir al enriquecimiento teórico (Jara, 2001). Estas son entonces las intenciones de este artículo, el cual representa a su vez una toma de conciencia de lo que ha sido RIFED, como ha evolucionado y hacia donde podría encaminarse. Para ello se parte de una contextualización integrado a una referente conceptual, una breve explicación metodológica para la sistematización lo cual conduce a establecer la evolución de RIFED, las fases por las que se ha desempeñado, para finalmente ofrecer una mirada en el horizonte, es decir los retos que asumir la Red en concordancia con su desarrollo y metas propuestas.

\footnotetext{
4 La Red Institucional de Formación y Evaluación Docente RIFED fue conceptualizada por las autoras de este artículo y la Dra. María Eugenia Venegas Renauld, ex Decana de la Facultad de Educación.
} 


\section{Marco contextual y conceptual}

Para entender cómo surge y se desarrolla RIFED, se ofrece de manera conjunta un marco contextual y de referencia ya que resulta difícil separar uno del otro, el contexto en el caso de RIFED, determina el marco conceptual.

\section{1 ¿Por qué una RED y no un Centro?}

\subsubsection{La propuesta del Consejo Universitario}

En el año 2005 el Consejo Universitario de la Universidad de Costa Rica solicitó a la administración la creación un Centro de Formación Continua del Profesorado fundamentado en tres ejes: formación en la disciplina, mediación pedagógica y cultura universitaria. Esta propuesta se justificó con las siguientes argumentaciones:

(...) la persona formadora debe responder, de manera efectiva, oportuna y creativa, no solo a las nuevas exigencias que impone el desarrollo científico, tecnológico, cultural, de nuevos paradigmas filosóficos y modelos socioeconómicos, sino, también, a los procesos de construcción y deconstrucción de marcos ideológicos en general y que, inevitablemente, se ven reflejados e influyen en nuestro entorno nacional, específicamente en los desafíos y oportunidades que la educación superior costarricense debe encarar con pertinencia en esta etapa contemporánea.Se ponen aquí en evidencia dos aspectos medulares:

a. Que la formación profesional y, en especial, la labor académica, no pueden ser consideradas de carácter "estático", como si fuese un producto concluido.

b. En consecuencia siendo el cuerpo docente universitario columna esencial del proceso enseñanza-aprendizaje, debe ser sujeto central de los esfuerzos institucionales, en pro del mejoramiento continuo de la calidad educativa superior (Consejo Universitario-UCR, 2005, pp. 2-3).

Ahora bien, aprobada esta propuesta, la responsabilidad de asumir esta tarea recayó, como corresponde, en la Vicerrectoría de Docencia. Siendo así, la señora Vicerrectora, Dra. Libia Herrero Uribe, conformó una comisión ad hoc para estudiar el proyecto en mención y proponer su puesta en marcha. Esta comisión, estando de acuerdo con las argumentaciones presentadas por el Consejo Universidad, señaló dos retos adicionales: 
1. ¿Cómo aprovechar la experiencia acumulada por la Universidad, durante más de treinta años, en materia de formación docente por parte de diversas instancias y personas?, y

2. La necesidad de incorporar en este proyecto el ámbito de la evaluación docente como una estrategia de mejoramiento e innovación de la docencia universitaria.

\subsubsection{RIFED como figura innovadora de gestión académica en la UCR}

Para dar respuesta a la solicitud del Consejo Universitario, así como a los retos planteados en el punto anterior y aprovechar además la experiencia acumulada por la Universidad en materia de redes académicas externas académicas desde 1982, es que la Vicerrectoría de Docencia propone la creación de la Red Institucional de Formación y Evaluación Docente (RIFED), en lugar de un Centro. De esta manera RIFED se constituye en la primera red de carácter institucional, que pretende establecer sinergias entre diversas instancias a lo interno de la Universidad.

La comisión convocada por la Vicerrectora de Docencia en el año 2006, encontró que la figura de red, resultaba ser la más conveniente para poder aprovechar la experiencia acumulada por la Universidad en materia de formación docente y permitiría además poner en relación dialógica los componentes de formación y evaluación docente, aprovechando además los recursos humanos y materiales existentes en las diversas instancias. Seleccionar la figura de red y no la de centro, daría la posibilidad de establecer, no un nuevo organismo centralizado, sino una nueva forma de comunicación y sinergia entre dependencias y personas (nodos).

En concordancia con lo anterior RIFED se inauguró en abril del año 2008 y:“(...) se concibe como un espacio de interacción institucional de naturaleza académica adscrita a la Vicerrectoría de Docencia, que atiende los ámbitos de educación continua, evaluación e investigación pedagógicas y mediación digital, en educación superior” (RIFED, 2007, p.13), cuyo propósito es: "Promover el desarrollo académico del profesorado universitario mediante una organización y estructura descentralizadas, que vinculan de manera sinérgica los esfuerzos, instancias, programas e iniciativas, así como los recursos que posee la Universidad de Costa Rica" (RIFED, 2007, p.13).

En concordancia con lo anterior, sus objetivos son: 
1. Promover en la comunidad académica universitaria, la reflexión sobre el desarrollo de la docencia universitaria, sus implicaciones pedagógicas en los procesos de aprendizaje, la evaluación y el desarrollo curricular.

2. Promover una cultura de evaluación para el mejoramiento del ejercicio docente.

3. Generar conocimiento pedagógico que sustente el quehacer docente, a través de la investigación y sistematización.

4. Generar experiencias de innovación pedagógica, mediadas por Tecnologías de la Información y comunicación (TICS). (RIFED, 2007, p.13)

Su estructura organizativa flexible y poco jerárquica articula acciones y recursos de las instancias actuales directamente involucradas en el desarrollo de procesos de formación pedagógica y curricular, evaluativa e investigativa de la docencia universitaria. Su estructura funcional promueve la vinculación sinérgica de funciones y logros, en los cuatro componentes que inicialmente fueron propuestos: desarrollo profesional, evaluación pedagógica, investigación pedagógica y mediación digital. (RIFED, 2007)

RIFED está conformada por todas las instancias y personas que se interesan y tienen funciones en la formación y la evaluación docente en la UCR. Para canalizar, apoyar y gestionar acciones y proyectos, cuenta con una Coordinación Ejecutiva, que se apoya en un Comité Ejecutor, un Comité Asesor, un Asesor en Comunicación y una Asistente de Gestión.

En este sentido RIFED es un organismo deslocalizado que funciona en una estructura localizada, la Universidad, caracterizada por una estructura jerárquica, vertical regulada por el Estatuto Orgánico y los reglamentos que de allí se derivan.

\subsubsection{El "Centro" como figura de gestión académica en la Universidad de Costa Rica}

El Tercer Congreso Universitario en 1973, ha marcado la estructura organizativa de la Universidad de Costa Rica hasta el día de hoy. Este congreso comprometió a la Universidad, no solo con el estudio y solución de los problemas nacionales, sino con la definición de una tendencia académica sobre otra meramente profesional, además se descentralizó la rectoría en las vicerrectorías. En este congreso se instaura el principio de indisolubilidad de la docencia, la investigación y la acción social, a razón de lo cual se propone la integración de las áreas de investigación y docencia, siendo este uno de los temas más discutidos; más sin embargo a pesar de este principio de indisolubilidad, el 
quehacer de las vicerrectorías correspondientes fue votado en funciones separadas en dicho Congreso. (Camacho, 1990)

El impulso a la investigación en este Tercer Congreso, es tal que la década de los 70 representa el período de mayor auge en cuanto a creación de centros de investigación en la Universidad, de los 25 existentes en la actualidad, al menos la mitad fueron creados en esa década 5 . Es así que el concepto de "Centro", como una de las figuras de gestión académica, se ve fortalecido desde esa época. Ello se ve reflejado en la estructura organizativa de la Vicerrectoría de Investigación: institutos, centros, laboratorios. Y en cada una de estas instancias se desarrollan programas y proyectos. En esta estructura también se cuenta con el Sistema de Estudios de Posgrado (SEP), el Sistema Editorial de Difusión Científica de la Investigación, el Sistema de Bibliotecas, Documentación e Información (SIBDI) y las Unidades Especiales de la Investigación (Estatuto Orgánico-UCR, 1974). Mas sin duda alguna la figura de centro ha sido la más utilizada en el quehacer investigativo.

Ahora bien, el caso de las Vicerrectorías de Acción Social, Vida Estudiantil y Docencia es diferente. La Vicerrectoría de Acción Social se organiza en secciones: Extensión Docente, Extensión Cultural, Trabajo Comunal, Administración y una Dirección de Gestión. La Vicerrectoría de Vida Estudiantil se organiza en oficinas de servicios (Registro, Becas, Salud y Orientación) y programas (voluntariado y liderazgo). Por su parte la Vicerrectoría de Docencia está integrada por un centro, una unidad y una red: Centro de Evaluación Académica (CEA), Unidad de Apoyo a la Docencia Mediada con TICS (METICS) y la Red Institucional de Formación y Evaluación Docente (RIFED). Valga señalar que el CEA y METICS, forman parte de RIFED. A lo interno de cada una de estas instancias se desarrollan proyectos, aunque de manera directa el personal docente puede inscribir proyectos de innovación docente de manera directa en la Vicerrectoría de Docencia.

Como se observa, la organización de cada Vicerrectoría ocurre de manera diferente, en este sentido se destacan dos aspectos, uno, que el elemento común corresponde al desarrollo de proyectos; y segundo que el concepto de Centro como figura de gestión

\footnotetext{
${ }^{5}$ La Universidad de Costa Rica, cuenta en el 2011 con 12 institutos y 25 centros de investigación, todos adscritos a la Vicerrectoría de Investigación, donde al menos catorce de ellos fueron creados en la década de los 70 . Solo un centro fue creado en la década de los 80 y otros diez centros han sido creados a lo largo de la década del 90 y hasta el 2008.
} 
académica, nace y se desarrolla casi de manera exclusiva en la Vicerrectoría de Investigación.

Desde el ámbito de la investigación, los Centros de Investigación tienen una estructura vertical: una dirección, una subdirección, un consejo asesor y un consejo científico. Se caracterizan por realizar su quehacer más en relación con instancias externas a la Universidad. Por su parte el Centro de Evaluación Académica, también cuenta con una dirección, sin embargo se organiza en secciones con sus jefaturas y su quehacer más bien se desarrolla a lo interno de la Universidad, en coordinación con las diversas unidades académicas.

En este contexto ¿qué es lo que distingue a un centro en el contexto de la Universidad de Costa Rica? Lo que encontramos es una estructura localizada, tanto en infraestructura como administrativamente, donde converge un quehacer particular; requiere además de su propia organización, centralizada y vertical, con sus propios recursos, tanto humanos como materiales. La toma de decisiones tiene un alto grado de independencia, es decir, aunque hay niveles de coordinación con otras instancias universitarias, la toma de decisiones finalmente recae en sus direcciones.

\subsubsection{El concepto de red}

Tal y como se señala en la introducción el concepto de red ha sido una de las inquietudes que han orientado el quehacer de RIFED, si bien inicialmente se parte de un concepto dado en el documento descriptivo sobre RIFED (2007), este concepto se ha ido retomando, por cuanto a nivel de gestión uno de los mayores retos ha sido esclarecer cómo se funciona una red, como instancia horizontal, dentro de una estructura institucional de carácter vertical. En razón de lo anterior y como resultado de la ruta de aprendizaje (ver apartado seis), el concepto de red se asume como un organismo deslocalizado.

Ya sea desde lo biológico o lo social, lo cierto es que un organismo tiene que ver con vida; necesariamente cuenta con una estructura compleja, en la cual interviene un sistema de comunicación y una organización que le permiten interactuar con el medio e intercambiar energía, materia o conocimientos. Esta interacción le posibilita el poder cumplir con las funciones de alimentarse, desarrollarse, relacionarse y reproducirse. Desde un punto de vista social, un organismo busca cumplir metas. 
Deslocalización implica una reorganización espacial. La deslocalización puede producirse en espacios distintos pero siempre bajo una misma finalidad o actividad, en este caso de carácter académico. En este sentido da cabida, no solo al aumento y diversidad académica, sino al mejoramiento.

Un organismo deslocalizado cuenta con una estructura organizativa descentralizada, que involucra diversos espacios, ámbitos y personas. Su acción ocurre de manera sincrónica o asincrónica y sus efectos se dan en variados espacios, no sólo en aquel donde se origina la acción, ya sea que ello ocurra de manera intencionada o no. Ese precisamente es el caso de las redes.

En concreto una red como organismo deslocalizado: "corresponde a un conjunto de elementos organizados interdependientemente con el fin de compartir recursos e información y potenciar funciones. Considera la existencia de las partes estrechamente vinculadas con sus funciones internas e influenciada por instancias externas" (RIFED, 2007, p.10). Como organismo vivo, la red activa nodos y utiliza diversos espacios porque requiere alimentarse, desarrollarse y evolucionar. Las decisiones son colectivas porque requiere energía e intercambiar conocimientos. De esta manera: "una de las principales funciones de las redes es contrarrestar la división del conocimiento en tres áreas del saber o culturas(...)la cultura científica, la de las humanidades y las ciencias sociales, así como establecer vínculos entre estas para construir conocimiento conjunto". (Viales, 2010, ๆ 4).

\subsection{5 ¿Cómo vive un organismo deslocalizado en una localidad?}

El concepto anterior nos lleva a la cuestión de cómo funciona una red (organismo deslocalizado) dentro de la institución (instancia localizada).

La deslocalización de un organismo puede darse en un ámbito espacial más o menos restringido, por ejemplo puede darse una deslocalización de una instancia a nivel de una institución o a nivel de varias instituciones, sean nacionales o internacionales, este también es el caso de las redes. En este sentido: “(...) las redes implican cooperación, pero también controversias y conflictos. En este sentido, las redes tienen sus propios procesos de evolución (Viales, 2010, ๆ 5).

Paradójicamente el organismo deslocalizado requiere de otros de carácter localizado. Su nivel de desarrollo está determinado por el sistema de comunicación que se implemente: de orientación más horizontal y colectiva. 
De tal manera que RIFED es un organismo deslocalizado que requiere de recursos localizados, por cuanto como red cuenta con pocos recursos propios. La diferencia está en identificar los recursos localizados en diferentes puntos institucionales, siendo el componente comunicativo el que genera sinergias para el logro de un objetivo común.

\subsection{6. ¿Para qué formar al personal docente universitario?}

Hoy por hoy esta pregunta es altamente significativa, no solo porque los contextos actuales demandan y presionan por una oferta universitaria de calidad y excelencia, sino porque en este marco de exigencias, la Universidad y los agentes que la conforman, se ven desafiados por nuevas tareas y funciones que requieren de algo más que solo información. La formación como proceso sistemático y sostenido brinda la oportunidad para desarrollar experiencias y capacidades que permiten a la comunidad universitaria ser proactivos en el cumplimiento de su misión.

No obstante, la formación no puede asumirse como un ejercicio vertical, el contexto actual y la complejidad que le caracteriza sugiere procesos de formación socialmente mediados: comunidades de personas que aprenden, que reflexionan acerca de sus prácticas y comparten los logros, además de evaluar las opciones de transformación y mejoramiento.

En la Universidad de Costa Rica la formación del personal docente tiene una historia de más de treinta años, su revisión deja entrever cómo ha evolucionado la noción de formación de su personal docente para proponer nuevas opciones de excelencia académica.

El III Congreso de la Universidad de Costa Rica marca, al igual que el caso de la evaluación el punto de partida para cambios estratégicos en materia de formación del personal docente universitario. En este Congreso, entre otros aspectos, se pusieron de manifiesto amplias discusiones entre lo académico y lo meramente profesional, así como las relaciones entre la investigación, la docencia y la acción social, para la formación del estudiantado (Camacho, 1999)

Es en el marco de los alcances de este congreso que se crea el Departamento de Docencia Universitaria (DEDUN), en el año 1975, como instancia de la Facultad de Educación, teniendo a su cargo: "(...) las acciones formativas de los docentes universitarios en materia pedagógica" (DEDUN, 2009, \1). Su base legal se expresa en el artículo 10 del Reglamento de Régimen Académico. En la actualidad el DEDUN asume labores de investigación y asesoría, con un equipo académico interdisciplinar. 
Desde su existencia, la Universidad se enfrenta al hecho de que su personal docente debe atender funciones docentes sin preparación para ello.

(...) la responsabilidad de la Universidad de Costa Rica en aras de promover acciones tendientes a la formación pedagógica del docente universitario va en estrecha concordancia con las necesidades emergentes de los docentes universitarios en un quehacer para el cual no fueron profesionalmente formados y además porque la educación superior costarricense, concebida como una institución con profundas relaciones con la sociedad, tiene un compromiso ético con el desarrollo de esta. La calidad de los procesos universitarios depende en primer término, de la calidad de los elementos del sistema entre ellos, la concepción pedagógica de los docentes misma que se asume como uno de los factores significativos (RIFED, 2007, p.37)

Es así como la Vicerrectoría de Docencia, encargada, entre otros asuntos de: "Velar porque la labor docente en la Universidad se lleve a cabo en forma eficiente y actualizada, en unidad de propósitos con la investigación, utilizando los sistemas más adecuados de enseñanza y evaluación" (Artículo 50, inciso ch. Estatuto Orgánico UCR. 1974, p.11), se ve llamada a desarrollar acciones estratégicas que lleven a una mayor cobertura, bajo un concepto de calidad, en la formación del profesorado universitario. Se crea de esta manera en el año 2007, la Unidad de Apoyo a la Docencia Mediada con Tecnologías de la Información y Comunicación (METICS) y en el 2008, la Red Institucional de Formación y Evaluación Docente,

La Unidad METICS, tiene como propósito el apoyo en la formación docente, centrada en la mediación didáctica con tecnologías para la información y comunicación (TICS). Cuenta para ello con un equipo humano multidisciplinar, para realizar labores transdisciplinares.

Es entonces en RIFED donde confluyen esfuerzos del CEA, el DEDUN, METICS y otras instancias con objetivos similares en cuanto a formación docente universitaria.

\subsection{7. ¿Para qué evaluar en la UCR?}

Para responder a esta pregunta, es necesario hacer referencia al contexto nacional, regional e internacional, siendo que para evaluar existen presiones externas e internas a la 
institución universitaria. También es necesario considerar que dadas estas presiones, en cada momento histórico las razones para evaluar han sido diferentes.

De la mano de Héctor Elí Rizo Moreno (2004) Vicerrector Académico de la Universidad Nacional de Colombia, haremos un recorrido histórico sobre las razones por las cuales se ha evaluado en las universidades latinoamericanas, en el último siglo. En plena Revolución Industrial se evaluaba para garantizar que las instituciones educativas formaran ciudadanos competentes en la repetición acertada de actividades; la memorización para poder seguir instrucciones contenidas en manuales; la disciplina y obediencia, pues la industria necesitaba personas que supieran acatar y someterse a las órdenes de sus superiores.

Bajo la influencia de Taylor y Fayol aparecen los términos de tecnología de la educación, diseño curricular, objetivos de aprendizaje o evaluación educativa, con un claro enfoque cuantitativo y una demarcación tecnológica de la evaluación. En los años treinta, de acuerdo con Rizo (2004) se presenta un estancamiento generalizado en la educación y la necesidad de optimizar los recursos dedicados al desarrollo de los programas educativos, por lo que Ralph Tyler propone la evaluación basada en objetivos, siempre con un enfoque cuantitativo, aunque se diversifican los contenidos por evaluar y se consideran las intenciones y los intereses de los propios protagonistas de los programas. Seguidamente, dice Rizo, en los sesenta, se incorpora la preocupación por la recolección y el uso de la información con fines utilitarios para la toma de decisiones y se establece una distinción entre evaluación formativa y evaluación sumativa.

En concordancia con el contexto histórico, económico y social resumido arriba, la evaluación del desempeño docente en la Universidad de Costa Rica, se inicia en la década de los 70 del siglo pasado, con un enfoque cuantitativo y una finalidad utilitaria: el ascenso en Régimen Académico Docente de su personal. El III Congreso Universitario (1972) dio paso a la creación del Centro de Evaluación Académica( $\underline{(E A)}$, una oficina coadyuvante de la Vicerrectoría de Docencia, a la cual se le encarga esta tarea.

Tal como explica la Licda. Lorena Kikut Valverde (entrevista radial, 17 setiembre, 2011) ${ }^{6}$, Jefa de la Sección Técnica de Evaluación Académica en esta evaluación se realiza desde ese momento y hasta la fecha, a partir de tres cuestionarios que se le aplican: 1) al estudiantado del o la docente que solicita evaluación de su desempeño con el propósito

\footnotetext{
${ }^{6}$ Para escuchar la entrevista completa a Kikut Valverde, descargar el podcast de Lenguajeos de la página www.catedrau.ucr.ac.cr
} 
mencionado; 2) a la Jefatura inmediata; al docente en cuestión. Los cuestionarios han sido elaborados siguiendo criterios científicos rigurosos; se han ido actualizando periódicamente y de acuerdo con Tyler (en Rizo, 2004) tomando en cuenta la opinión de los propios protagonistas; tienen validez y confiabilidad; y están a disposición del profesorado para que pueda revisarlos en cualquier momento. En el transcurso de todos estos años, esta evaluación ha permitido conocer las fortalezas y debilidades del personal docente de la institución, con miras a la toma de decisiones y le ha permitido al profesorado avanzar en su carrera académica de acuerdo con lo que establece para tal efecto el Reglamento de Régimen Académico y Servicio Docente de la Universidad ${ }^{7}$.

Sin embargo, tal como reconoce Kikut (entrevista radial, 17 setiembre, 2011), este tipo de evaluación (como todos los tipos) tiene limitaciones. Por ejemplo, el profesorado se queja de que, al ser un instrumento cuantitativo y tipo encuesta, las preguntas permiten reflejar las innovaciones docentes y las mejoras cualitativas que realizan en sus clases.

Regresando al contexto histórico la llamada globalización, el avance de la ciencia y el desarrollo de nuevas tecnologías digitales abren nuevas perspectivas para los cambios ideológicos de las organizaciones y de la sociedad (Rizo Moreno, 2004). La experticia que más se valora en personas es su creatividad y la educación debe contribuir a desarrollar las máximas intelectuales que un ser humano puede desarrollar. Se habla de una economía del conocimiento y los llamados hechos económicos asociados a la producción, el consumo y el intercambio quedan circunscritos tanto a la creación de conocimiento como a la preferencia, a la utilidad del nuevo conocimiento y a la imperfección de su mercado (Rizo Moreno, 2004). En este contexto, de acuerdo con el autor, se impulsan, desde las esferas gubernamentales, esquemas de rendición de cuentas; ejercicios de autoevaluación con perspectivas de acreditación o certificación.

En este nuevo panorama, la Universidad de Costa Rica acredita su primera carrera en el año 2001 y a partir de entonces, cada unidad académica que se involucra en procesos de autoevaluación con miras a la acreditación, incorpora la evaluación sistemática de sus docentes. Esta evaluación es independiente de la mencionada anteriormente; cada unidad académica diseña sus propios instrumentos y puede solicitar apoyo al Centro de Evaluación Académica si lo considera oportuno. Aún en este contexto y con las diferencias señaladas, la

7 ver http://www.cu.ucr.ac.cr/normativ/regimen academico docente.pdf 
evaluación continúa siendo cuantitativa y reporta los beneficios y las limitaciones comentadas anteriormente.

A partir del año 2005 y sobre todo a partir de la creación y el apoyo de $\underline{\text { RIFED }}$, se han introducido algunas mejoras a estos procesos de evaluación cuantitativa, tanto para ascenso en Régimen Académico, como en el marco de los procesos de autoevaluación de las unidades académicas:

- Por indicación de la Dra. Libia Herrero Uribe, Vicerrectora de Docencia, al profesorado que obtenga calificaciones deficientes en su desempeño docente se le ofrecen alternativas para superar sus debilidades, tanto por parte del Departamento de Docencia Universitaria DEDUN, como por parte de RIFED.

- De igual manera, por indicación de la Dra. Herrero, este mismo profesorado deberá ser nuevamente evaluado en el ciclo lectivo subsiguiente, para revelar la mejora que evidencia en su desempeño docente o tomar otras decisiones pertinentes de acuerdo con los resultados obtenidos en esta segunda evaluación.

- Se ha desarrollado una aplicación digital de los cuestionarios de manera que el estudiantado, la jefatura y la propia persona docente puede contestarlos independientemente de su ubicación física o temporal, con el consecuente ahorro de papel y tiempo en el procesamiento de los datos.

- Se ha introducido la tecnología conocida como "clickers", un sistema de votación electrónico, que arroja los resultados de manera instantánea, también con el consecuente ahorro en papel y tiempo.

- El estudiantado ha respondido muy favorablemente a estas dos últimas mejoras. (Kikut, entrevista radial, 17 setiembre2011).

\section{Abordaje metodológico para la sistematización}

El desarrollo metodológico para la sistematización ha estado caracterizado por una reflexión durante el proceso. Es decir desde la creación de RIFED en el año 2008, su comité ejecutor ha prestado atención a la forma como se va desarrollando la Red, los cambios conceptuales y de gestión que van dando, con la intención de alcanzar sus metas. Es así que desde un inicio se han establecido tres inquietudes que ha servido de referente para la reflexión y la toma de decisiones de manera recurrente: qué es una red, cómo funciona una 
red académica y como debería funcionar RIFED. Adicionalmente se han utilizado como insumos y forma de organizar la información (ver Tabla 1), la siguiente documentación:

- Documento descriptivo sobre RIFED (2007), el cual se constituye en un punto de referencia conceptual y de gestión.

- Minutas tomadas en cada reunión para el período 2008-2011, las cuales han servido para dar seguimiento a los diversos acuerdos, actividades, establecer propuestas, además como insumo esencial para la elaboración de informes.

- Informes de labores presentados durante cada año, se han constituido en insumo para la reflexión, replanteamientos conceptuales y toma de decisiones.

- Informe trienal para el período 2008-2010. Muy en especial este informe ha servido para replanteamientos teóricos y de gestión, por cuanto considera a los otros informes de actividades y evaluaciones.

- Informes de actividades y evaluaciones. Han permitido, no sólo valorar logros sino también procedimientos y especialmente las conceptualizaciones por cuanto se obtienen valoraciones por parte de las personas involucradas. Estas evaluaciones e informes también han sido insumo para la elaboración de informes.

Tabla 1

Cantidad de documentos utilizados para como insumo para la valoración del desarrollo de RIFED

\begin{tabular}{|c|c|c|c|c|}
\hline $\begin{array}{c}\text { Años de } \\
\text { gestión }\end{array}$ & \multicolumn{4}{|c|}{ Cantidad documentos sobre la gestión de RIFED } \\
\hline & Minutas & $\begin{array}{c}\text { Informes de } \\
\text { labores }\end{array}$ & $\begin{array}{c}\text { Informes de } \\
\text { actividades }\end{array}$ & $\begin{array}{c}\text { Informes de } \\
\text { evaluación }\end{array}$ \\
\hline 2008 & 12 & 1 & 1 & - \\
\hline 2009 & 7 & 1 & - & - \\
\hline 2010 & 10 & 1 (trienal) & - & - \\
\hline 2011 & 10 & - & 1 & 6 \\
\hline Total & 39 & 3 & 2 & 6 \\
\hline
\end{tabular}

Fuente: Información ofrecida por Stephanie Fallas. Oficina de apoyo de RIFED. Vicerrectoría de Docencia.

Cada informe de labores ha representado una sistematización parcial sobre la evolución de RIFED. Más sin embargo es en el apartado cinco, sobre la "Ruta de Aprendizaje" donde se describe y explica la evolución que ha tenido RIFED. Los apartados seis a siete, señalan aspectos conceptuales derivados de la sistematización y el apartado 
ocho presenta una visión prospectiva del quehacer de RIFED, derivada también de esta sistematización.

\section{Ruta de aprendizaje de RIFED}

Esta ruta de aprendizaje representa la sistematización sobre la evolución que ha tenido RIFED a lo largo de cuatro años de coexistir en el entorno universitario, ha implicado un proceso de aprendizaje difícil e inacabado. ¿Cómo definir funciones, cómo lograr sinergias, cómo entender la horizontalidad de la comunicación, cómo trabajar en colectivo, como identificar nodos y cómo activarlos, en concreto, cómo trabajar en red? Esos han sido los desafíos en RIFED, cuya ruta de aprendizaje se puede determinar hasta el momento en varias fases (RIFED, 2010): inicialmente la inserción en el ámbito universitario por medio de la divulgación, luego una aproximación al trabajo en red para posteriormente llegar a un momento de activación, lo cual ha permitido la generación de nuevas conceptualizaciones.

\subsection{Inserción en el ámbito universitario: la divulgación}

Dado que RIFED es un organismo deslocalizado: "su inserción en la comunidad universitaria requirió de una labor importante de divulgación masiva, con el fin de diagnosticar el escenario donde se gestaría la Red y apoyar asertivamente sus primeras acciones" (RIFED, 2010, pp.15-16). Es así como esta fase estuvo caracterizada por cuatro tipos de acciones:

Presentaciones a lo interno de la Universidad: correspondieron espacios informativos sobre las intenciones y forma de organización de la Red. Se realizaron presentaciones ante el Consejo Universitario, direcciones de unidades académicas, jefaturas de oficinas, destacan las once presentaciones ante asambleas de Facultad y Sedes Regionales, así como el acto inaugural que permitió presentar la Red ante la comunidad universitaria en general, el día 10 de abril del 2008.

"Este acercamiento a las unidades académicas permitió obtener algunas percepciones en torno a las necesidades de formación y evaluación del docente universitario, así como las expectativas alrededor de la creación de esta Red" (RIFED, 2010, p. 21. Adicionalmente estos espacios sirvieron para que algunos docentes ofrecieran su apoyo a algunas iniciativas que empezaban a organizarse. 
Presentaciones a lo externo de la Universidad: estas presentaciones, similares a las anteriores, sirvieron de complemento, por cuanto se realizaron a nivel externo (nacional o internacionalmente), en congresos, simposios, conferencias, jornadas y otros espacios académicos. Destaca la presentación en un taller organizado por GUCAL ${ }^{8}$. Estas presentaciones permitieron identificar contactos para futuras actividades.

Diseño de un elementos gráficos y digitales: se logró el diseño de un portal web http://rifed.ucr.ac.cr, un logo, un desplegable, así como la asignación de una cuenta electrónica rifed@ucr.ac.cr y la apertura en un espacio en la red social facebook.

Jornada de reflexión: Esta actividad tuvo como objetivo valorar el quehacer de cada instancia (Departamento de Docencia Universitaria, Centro de Evaluación Académica y CEA y Unidad METICS), dentro de la dinámica de RIFED. También fue un espacio para visualizar proyectos y acciones, de trabajo conjunto, por ofrecer a la comunidad universitaria, acordes con los objetivos de la Red.

\subsection{Aproximación al trabajo en Red}

En esta fase se ha trabajado en dos ámbitos, uno a nivel gestión y el otro sobre el tipo de acciones por desarrollar. A nivel de gestión se trabajó en varias líneas con la intención de lograr una aprehensión y apropiación del trabajo en red, a saber:

- Niveles y formas de comunicación: la comunicación entre los miembros de la comisión ejecutora, de esta con el comité asesor, y con la coordinación de RIFED, con los docentes y unidades académicas.

- Registro del quehacer de RIFED. Definición de actas e informes.

- Esclarecimiento de funciones: por parte de las coordinaciones de cada componente y la coordinación y el personal de apoyo.

- Interrelación entre componentes.

${ }^{8}$ El Grupo Universitario para la Calidad de América Latina (GUCAL) fue creado en el 2005 y está integrado por personas egresadas de programas de la cooperación alemana en el campo de la gestión de la calidad y el cambio universitario, específicamente de University Staff Development Programme (UNISTAFF), Programa Internacional de la Gestión de la Calidad y del Cambio en la Educación Superior (UniCambio XXI), Curso de Capacitación en Métodos Innovativos de Autoevaluación (Camina 2001). 
- Manejo de resistencias a nivel interno: Sesiones de reflexión sobre las implicaciones del trabajo en red, lo cual requirió lecturas reordenación de procedimientos, para tratar de trascender hacia una forma de trabajo descentralizada y deslocalizada, propia de una red.

Sobre el tipo de proyectos y acciones por desarrollar. Con la intención de abordar el trabajo en red de manera más coherente, durante el año 2009 y hasta mediados del 2010, se establecieron cinco dimensiones estratégicas para los cuatro componentes de RIFED (formación, evaluación, investigación, mediación digital), a saber:

- Formación. Su objetivo es generar acciones de formación sistemática para el profesorado universitario que permita fortalecer su desempeño académico.

- Reflexión. Su objetivo es desarrollar una cultura de reflexión, mediante actividades abiertas y de autoformación en temas relacionados con la formación y la evaluación docente en la educación superior, la participación en redes y el funcionamiento de RIFED.

- Investigación. Su objetivo es establecer el estado de la cuestión sobre la investigación pedagógica en la Universidad de Costa Rica.

- Difusión y Divulgación. Su objetivo es disponer de mecanismos y recursos, sean impresos, cibernéticos u otros, para divulgar el quehacer de RIFED, así como difundir el conocimiento, reflexión e intercambio de experiencias académicas en el ámbito de la didáctica, evaluación, innovación y uso de las TICS en la docencia universitaria.

- Producción. Su objetivo es la elaboración de publicaciones, materiales y recursos sean impresos, cibernéticos u otros, para fortalecer los procesos de formación y evaluación del docente universitario.

Durante esta fase de aproximación al trabajo en red (año 2009) se desarrollaron proyectos y actividades, (ver recuadro 1) alcanzó una participación de 3,625 miembros, lo cual contribuyó a configurar nuevas formas de trabajo y de comunicación para RIFED. A partir de II semestre del año 2010 se inició una serie de ajustes para alcanzar una maximización del potencial de recursos disponibles e incorporar nuevos roles en los miembros de la red para atender la actual y futura demanda (RIFED, 2010) 


\subsection{Activación de la Red}

Con una mayor claridad sobre las funciones y la forma de comunicación, para el segundo ciclo del 2010 se logra una mejor interrelación e integración de los componentes de la red. En este sentido se propicia la gestión y acompañamiento de programas, proyectos e iniciativas que facilitan el establecimiento de enlaces estratégicos para el desarrollo de diferentes espacios de interacción de los miembros, con miras a una mayor sostenibilidad (RIFED, 2010).

Para este momento se observa una mayor madurez en el trabajo en red. Siempre en concordancia con las dimensiones estratégicas señaladas en la fase anterior, se establecen particularmente cuatro tipos de acciones: las visitas académicas las tertulias de autoformación, las pasantías y jornadas de actualización con unidades académicas.

Recuadro No. 2

Visitantes académicos

- Dr. Eric Mazur Profesor de Física de la Universidad de Harvard.

- Master Angélica Natera del Programa LASPAU

- Dra. Alicia Camilloni y el Dr. Augusto Pérez de la Universidad de Buenos Aires, Argentina.

- Dr. Walter Bender, Director de Sugar Labs, OLPS association. Dra. Claudia Urrea, Directora Educativa de Asociación OLPC.

Fuente: Informe de Labores 2008-2010. RIFED.

Visitas académicas: por parte de académicos de reconocido aporte pedagógico (ver recuadro 2).

Las tertulias de autoformación: espacios conducidos por los mismos docentes, con participación de sus pares. En total 16, con las siguientes temáticas: 
Pasantías: Espacios de actualización y reflexión sobre la función docente. Las personas participantes se comprometen a realizar algún proyecto a nivel institucional. En coordinación

Recuadro No. 3

Tertulias de autoformación

- ¿Cómo hacer clases interesantes?

- Repensar la docencia: una experiencia de mediación virtual

- Autogestionar la innovación curricular en la educación superior

- Conocer al otro: desafíos y oportunidades en la relación estudiante-docente

- ¿Cómo aprovechar la evaluación del desempeño para mejorar la función docente?

- Aprendizaje y Creatividad

- Habilidades Comunicativas en la Educación Superior

- Docencia e Investigación

- . Desarrollo de materiales Hipermediales.

- Innovación docente: Profe, ¿usted cree que está innovando?

Fuente: Informe de Labores 2008-2010. RIFED. con LASPAU, Vicerrectoría de Docencia y la Oficina de Asuntos Internacionales, hasta el momento se han organizado dos promociones de pasantías a la Universidad Harvard, una en el 2010 y otra en el 2011, en ambas ocasiones para un grupo de 12 docentes.

Jornadas de actualización con unidades académicas: Corresponden a actividades más integradoras por cuanto se trabaja con el personal docente de unidades académicas, hasta el momento a petición de la Escuela de Estudios Generales y la Escuela de Administración de Negocios.

\section{Nuevas funciones}

Vivir en forma diferente supone vivir nuevas experiencias, y por lo tanto, reconsiderar nuestras experiencias y decisiones. Aun más vivir en forma diferente en una localidad que conserva las prácticas cotidianas regulares, algunas de ellas tradicionales, otras más diversas, supone pensar las nuevas experiencias y decisiones a partir de nuevos roles. La ruta de aprendizaje de RIFED necesitó recrear las funciones que los académicos desarrollamos en el ámbito universitario, las cuales se describen a continuación.

\subsection{De lo vertical a lo horizontal}

Establecer acciones en red implica reconocer que todas las personas que la constituyen ofrecen aportes y expectativas, de tal manera que para lograr su articulación, la horizontalidad será la plataforma de intercambio. Las universidades como organizaciones que datan de la Edad Media, han estado caracterizadas por relaciones jerárquicas asociadas al manejo del poder formalizado. Las redes aprovechan las capacidades y especialidades 
de cada quien para poder actuar, sobre la base de que todos y todas somos necesarios, de ahí que las jerarquías se flexibilizan y se dinamizan en el tiempo: los papeles de la experticia son intercambiables en el tiempo y en el espacio.

\subsection{Académicos y académicas aprendiendo}

La Red como construcción organizativa también nos dirigió hacia la necesidad de ver el aprendizaje como proceso activo, compartido e inacabado. En efecto, para vivir la experiencia de RIFED se hace necesario reconocer que quienes la conformamos tenemos diversas experiencias vitales, que una vez compartidas entre pares, son fuente de nuevos aprendizajes. Recordemos que transitamos en una instancia, la universidad, en la cual el saber, la experticia y la academia implican dominio, el trabajo en red relativiza ese dominio al distribuir los aprendizajes de las personas que la conforman.

\subsection{Aprender para formar nuevos roles}

Esta nueva experiencia, del trabajo en RIFED, permite ampliar los espacios para el aprendizaje, que tal y como indica Bain (2006) es natural y crítico. Natural porque se aprende de las prácticas y reflexiones cotidianas del docente universitario, de lo que es auténtico en la acción académica - universitaria, es un aprendizaje que despierta interés, curiosidad, es "intrínsecamente interesante" y crítico porque se reflexiona sobre lo compartido, se mediatiza entre los diferentes niveles de experticia que los miembros de la red tenemos. Reconocer la experticia distribuida nos orienta a nuevos roles: promotores, motivadores, expertos, aprendices, entre otros, todos intercambiables según las experiencias que la red va proponiendo.

\section{Fortalecimiento de la red}

Paralelo al posicionamiento de la red como espacio de aprendizaje y formación fue necesario también aprender a vivir en red. El fortalecimiento de la red no solo reconoció la necesidad de invitar a nuevos participantes a la red, sino además, que quienes la conformamos nos convertimos en aprendices de una nueva forma de trabajo y de intercambio académico.

Los docentes que se han ido sumando a RIFED han desarrollado y compartido nuevas estructuras de relación para su formación (autoformación y ecoformación), adicionalmente 
han establecido procesos de reflexión acerca de su propia docencia, lo cual les permite valorar su crecimiento en espacios de cooperación y colaboración.

El desarrollo de diversas actividades sobre formación y evaluación: tertulias, conversatorios, conferencias, encuentros de innovación, entre otras, han traído consigo el fortalecimiento de interacciones entre las personas participantes, a su vez han servido para gestar nuevas relaciones que en el transcurso del tiempo se han fortalecido, una vez que se ha destacado la naturaleza del aprendizaje natural y crítico de quienes participan en la Red.

\section{Nuevas conceptualizaciones: ¿Cuál formación y cuál evaluación docente?}

Como ya se indicó la formación y evaluación docente tiene su recorrido histórico en la Universidad de Costa Rica que data de más de treinta años. Durante este tiempo las actividades crearon diversas formas de pensar y de concebir estos procesos los cuales también debieron ser considerados en el proceso de aprender a ser RIFED. El proceso histórico generó creencias y formas de expresión y representación que redujeron la formación y la evaluación a cierto tipo de prácticas, no siempre adecuadas, o bien, no comprendidas por las audiencias académicas, por ello era común escuchar frases como: lo educativo no sirve, los métodos y las técnicas didácticas son "recetas" o instrucciones, la evaluación es hacer exámenes, y otras. Estas creencias podían minar la posibilidad de innovar y recrear estos procesos. Así el proceso de construir la RED tuvo que enfrentar también la modulación y reconfiguración de expectativas, a fin de establecer una cultura de autoaprendizaje y ecoformación que transformara a su vez, las nociones de formación y evaluación del personal docente universitario.

\subsection{Sobre la formación docente}

La palabra formación se asocia con el proceso educativo (García y García, 1996) de ahí que cuando hablamos de formación en sí misma se alude a un proceso de superación, el cual es inacabado y por tanto histórico. En este marco, la formación permite crear, recrear y renovar la cultura y sus prácticas, por ello, el acto formativo nos lleva a transformar las realidades, a partir de finalidades conscientes libremente elegidas (Yurén, 2000).

Este concepto supone que quienes asumimos la tarea docente recreamos realidades mediadas por procesos reflexivos que nos permiten transformarlas, dejamos de pensar que la formación es acumulación de información y conocimientos indispensables para enseñar y 
asumimos que es aquella "capacidad de poder pensar también en los puntos de vista de los demás" (Gadamer, citado por García, 2006, p.20).

Así, desde este punto de vista, el personal docente universitario tiene la oportunidad de asumirse en una comunidad que intercambia sus experiencias vitales y académicas, las piensa y recrea en espacios que dan la oportunidad de compartirlas y reflexionarlas. Es un concepto de formación docente que considera los trayectos históricos de todos y todas, que les recrea un espacio para potenciar y crecer en la capacidad docente.

RIFED se basa en la idea de que toda experiencia mediada por la reflexión de quienes conformamos la comunidad académica, contribuye con el proceso de superación docente, fortalece las capacidades de interacción y cooperación entre redes de aprendizaje: "El modo de ser de la cosa se nos revela hablando de ella" (Gadamer, citado por García, 2006, p. 33).

Es por ello que en el marco de RIFED se han desarrollado y promovido diversas experiencias formales en las que el basamento de acción es la comunicación entre pares, la colaboración mutua entre quienes desarrollamos las tareas docentes y académicas y las redes de aprendizaje. Ejemplos de lo anterior son tertulias y conferencias por un lado y por otro, formalmente aprovechando la localización académica del DEDUN y su relación con diferentes instancias universitarias como el CEA, METIC, el Sistema de Bibliotecas, Documentación e Información (SIBDI), Comunidad de Software Libre UCR y Vicerrectorías, entre otras, los programas de formación a nivel del Licenciatura en Docencia Universitaria y en el marco del Programa de Posgrado de Ciencias de la Educación de la Facultad de Educación la Maestría en Educación con énfasis en Docencia Universitaria.

\subsection{Sobre la evaluación docente: hacia una evaluación orgánica sobre el desempeño docente}

Ya en pleno siglo XXI, la Universidad debe incorporar una nueva concepción de la evaluación docente, que incluya pero trascienda la evaluación cuantitativa explicada en el apartado sobre marco contextual y conceptual. Tal como indica el diccionario de la Real Academia de la Lengua, (2011) a evaluación es una acción de estimar, de apreciar, de señalar el valor. Por lo tanto, la evaluación del desempeño docente debe realizarse en primer lugar para que promueva una reflexión sobre la propia docencia y como una consecuencia directa, un mejoramiento permanente de la misma. 
En el pasado, la evaluación del desempeño docente ha estado desligada del aprendizaje del estudiantado y (tal como lo ha manifestado el profesorado de la institución) desarticulada de las demás tareas académicas del profesorado: la investigación y la acción social.

Las más recientes investigaciones (Murillo, et al, 2007) concuerdan en que la evaluación del desempeño docente es una condición fundamental para mejorar el aprendizaje del estudiantado; para atraer a los mejores candidatos hacia la profesión docente y mantenerlos motivados hasta su jubilación, así como para elevar la calidad de la docencia.

En RIFED creemos también que la evaluación del desempeño docente, debe ser un proceso permanente interconectado no solamente con el aprendizaje del estudiantado, sino con el aprendizaje del propio profesorado en relación con su quehacer pedagógico. De igual forma sabemos que debe estar interrelacionado con la investigación y la acción social y que estas tres acciones sustantivas de la academia deben impactarse mutuamente. En ese sentido consideramos la evaluación docente como un organismo vivo, e interdependiente, que como dijimos anteriormente incluye, pero trasciende la evaluación cuantitativa.

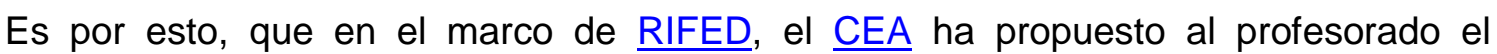
Portafolio de Vida Docente (PVD). Tal como explica la Licda. Elsiana Guido Guido (entrevista radial, 24 de setiembre, 2011) ${ }^{9}$, investigadora del Departamento de Investigación y Evaluación Académica DIEA del CEA, el PVD es en primera instancia una herramienta de autoevaluación docente. Permite prioritariamente al profesor o profesora reflexionar sobre su propio quehacer, buscar las instancias y estrategias que le permitan mejorar o innovar, y plantearse su propia ruta para hacerlo.

De igual forma, es una herramienta que facilita la reflexión para interrelacionar las actividades de docencia, investigación y acción social, buscando la manera de que tengan un impacto mutuo. El PVD, como su nombre lo indica, debe acompañar al profesorado durante toda su vida académica, pues la reflexión sobre la propia práctica es una condición inherente a la función docente.

Además, el PVD también puede convertirse en un instrumento para ascenso en Régimen Académico, si el docente establece una correlación entre su propio aprendizaje y el

\footnotetext{
9 Para escuchar la entrevista completa a Elsiana Guido, se puede descargar el podcast con el programa Lenguajeos, de www.catedrau.ucr.ac.cr
} 
de sus estudiantes; si revela la coherencia entre sus creencias sobre la docencia universitaria y sus acciones en el aula, y si sus planes de mejora o innovación han derivado de sus reflexiones y los resultados de la evaluación cuantitativa, y lo somete a valoración por parte de pares.

Es de esta forma, poniendo a disposición del profesorado herramientas de evaluación y autoevaluación cuantitativas y cualitativas que se interrelacionan entre sí, que RIFED lleva a la práctica su visión de una evaluación docente orgánica cuyo fin último es la excelencia en la docencia universitaria.

\section{8. ¿Cómo se mira RIFED en el horizonte?}

Horizonte (del griego opí̧ovtas "orizonta": limitar) es la línea que aparentemente separa el cielo y la Tierra. Vista desde cualquier ángulo esta línea siempre aparece a la altura de los ojos del espectador. Es saber popular, que el horizonte es inalcanzable: mientras más se avance hacia allá, más se aleja y mantiene intacta la distancia que nos separa (Wikipedia 2011).

En RIFED no nos planteamos una meta, sino un horizonte. Queremos tenerlo siempre visible y aunque sabemos que no se alcanza, si hemos comprobado como las ideas allí presentes pueden hacerse realidad, asumiendo los retos contenidos en ese horizonte.

La presentación de realizaciones que hemos hecho en este artículo ocurrió en momentos de la conceptualización de $\underline{\text { RIFED, }}$, retos e ideas que se asomaban en el horizonte. Al avanzar hacia estas ideas y al asumir los desafíos, muchos de ellos se fueron cristalizando y convirtiéndose en realizaciones. El horizonte, por el contrario, se alejó y (siempre visible), lo cual nos recuerda aquellas ideas y retos que no cristalizaron totalmente. Pero adicionalmente nos presenta nuevos desafíos:

- Una Red Institucional de Formación y Evaluación Docente fortalecida, con un número creciente de nodos (instancias y personas) participando activamente en procesos de auto y mutua capacitación los temas de formación y evaluación docente universitarias.

- Una comunidad académica con la flexibilidad de colaborar, aprender y mediar en el aprendizaje de otros, en un contexto deslocalizado.

- Una RIFED sometida voluntariamente a un proceso de autoevaluación permanente para actualizar sus referentes teóricos y valorar la pertinencia y eficacia de algunas estrategias de formación (tertulias de autoformación, conversatorios, programas 
específicos, pasantías) y evaluación docente (PVD); así como para proponer mejoras e innovaciones.

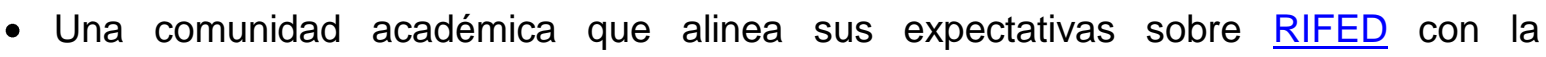
organización descentralizada y deslocalizada de la red.

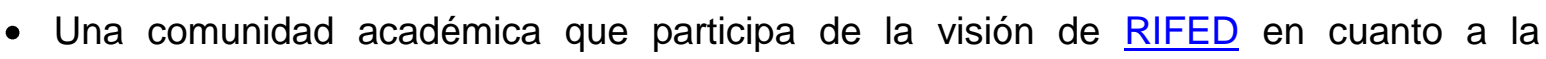
formación y la evaluación docente, necesarias en el momento histórico actual; o que propone visiones complementarias o alternativas debidamente fundamentadas.

- Acciones concretas que permitan visualizar el mutuo impacto de la docencia, la investigación y la acción social.

- Mayor cantidad de investigaciones sobre la formación y la evaluación docente universitaria.

- Acciones concretas que permitan evidenciar la relación existente entre formación y evaluación del profesorado universitario.

- Acciones académicas concretas que permiten visualizar el mutuo impacto entre el aprendizaje docente y el aprendizaje del estudiantado.

- Acciones académicas concretas que permitan visualizar interrelación entre distintas sedes de la UCR, así como entre distintas facultades, escuelas, áreas y disciplinas.

Al ir logrando estos desafíos, de manera continua se irán visualizando en el horizonte retos emergentes e ideas innovadoras que serán necesarias de atender. Ello implica que RIFED, como organismo deslocalizado, debe mantenerse en dinámica permanente de transformación, en coherencia con su marco conceptual de referencia, la cultura institucional y el horizonte que emerge. 


\section{Referencias}

Bain, Ken. (2006). Lo que hacen los mejores profesores universitarios. España: Universitat de Valencia.

Camacho Monge, Daniel. (1990). La inacabada lucha pro-académica del III Congreso Universitario. Revista De Ciencias Sociales, (49-50), 63-76.

Ferreiro Jesús, Gómez Carmen y Rodríguez Carlos. (2008). Deslocalización e inversión extranjera directa. Incidencia en la Economía Vasca. Revista de Estudios Empresariales. Segunda época, 1, 21-40.

García Carrasco, Joaquín., y García del Lujo, Ángel. (1996). Teoría de la Educación I Educación y Acción pedagógica. Salamanca: Ediciones Universidad Salamanca.

García Perea, María Dolores. (2006). Formación: Concepto vitalizado por Gadamer. México: Castellanos Editores.

Guido Guido, Elsiana (2011, 24 de setiembre). El Portafolio de Vida Docente en la Universidad de Costa Rica. Entrevista en programaradial Lenguajeos: Creatividad en Innovación en la Docencia. Vicerrectoría de Docencia. Universidad de Costa Rica. Podcast en www.catedrau.ucr.ac.cr .

Jara Holliday, Oscar. (2001, abril). Dilemas y Desafíos de la Sistematización de Experiencias. Ponencia presentada en el Seminario ASOCAM: Agricultura Sostenible Campesina de Montaña, Cochabamba, Bolivia. En http://www.alboan.org/archivos/335.pdf

Kikut Valverde, Lorena. (2011, 17 setiembre). Evaluación Docente en la Universidad de Costa Rica. Entrevista en programaradial Lenguajeos: Creatividad en Innovación en la Docencia. Vicerrectoría de Docencia. Universidad de Costa Rica. Podcast en www.catedrau.ucr.ac.cr

Murillo Torrecilla, Javier F.; González de Alba, Verónica y Rizo Moreno, Hector. (2007). Evaluación del Desempeño y Carrera Profesional Docente: Un estudio comparado entre 50 países de América Latina y Europa, Oficina Regional de Educación de la UNESCO para América Latina y el Caribe OREALC/UNESCO Santiago. Recuperado de http://unesdoc.unesco.org/images/0015/001529/152934s.pdf

Real Academia Española. (2011). Diccionario de la lengua española (22 ${ }^{\mathrm{a}}$ ed.). Recuperado de www.rae.es

Red Institucional de Formación y Evaluación Docente. (2007). Documento Descriptivo. San José, C.R.: RIFED, Vicerrectoría de Docencia, Universidad de Costa Rica

Red Institucional de Formación y Evaluación Docente. (2010). Informe de labores 20082010. San José, C.R.: RIFED, Vicerrectoría de Docencia, Universidad de Costa Rica. 
Rizo Moreno, Héctor Eli. (2004).Evaluación del Docente Universitario, una visión institucional, en Revista Iberoamericana de Educación, recuperada el 06 de agosto de 2011 de http://www.rieoei.org/deloslectores/883Rizo.pdf.

Ruiz Botero, Luz. (2001). La sistematización de prácticas. Sistematización de experiencia de convivencia. Liceo Nacional Marco Fidel Suárez. Recuperado de http://www.oei.es/equidad/liceo.PDF

Universidad de Costa Rica. (s.f.). Estatuto Orgánico de la Universidad de Costa Rica. Recuperado de http://www.cu.ucr.ac.cr/normativ/estatuto organico.pdf

Universidad de Costa Rica. Departamento de Docencia Universitaria. (2009). Página web DEDUN. Recuperado de http://docenciauniversitaria.ucr.ac.cr/

Universidad de Costa Rica, Consejo Universitario, 2011. Reglamento de Régimen Académico y Servicio Docente.www.cu.ucr.a.cr

Viales Hurtado, Ronny. (15 de julio 2010). Redes promueven desarrollo Académico. Recuperado de Portal de la Investigación de la Universidad de Costa Rica http://www.vinv.ucr.ac.cr/index.php?option=com content\&view=article\&id=498:redespromueven-desarrollo-academico-e-investigacion\&catid=1\&ltemid=68

Yurén C., María Teresa. (2000). Formación y puesta a distancia: su dimensión ética. México: Paidós.

Wikipedia. (2011). Horizonte. Recuperado de http://es.wikipedia.org/wiki/Horizonte 